\title{
Level sets as progressing waves: an example for wake-free waves in every dimension
}

\section{Wolfgang Quapp \& Josep Maria Bofill}

\section{Journal of Mathematical Chemistry}

ISSN 0259-9791

Volume 52

Number 2

J Math Chem (2014) 52:654-664

DOI 10.1007/s10910-013-0286-9

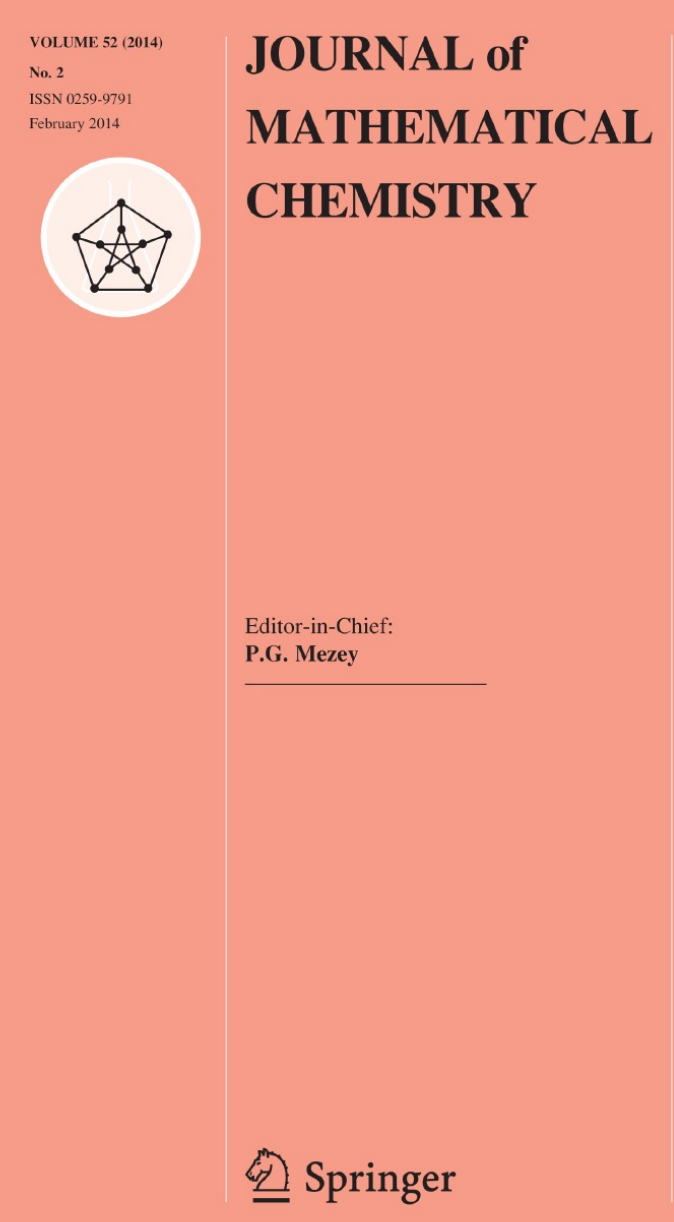

Springer 
Your article is protected by copyright and all rights are held exclusively by Springer Science +Business Media New York. This e-offprint is for personal use only and shall not be selfarchived in electronic repositories. If you wish to self-archive your article, please use the accepted manuscript version for posting on your own website. You may further deposit the accepted manuscript version in any repository, provided it is only made publicly available 12 months after official publication or later and provided acknowledgement is given to the original source of publication and a link is inserted to the published article on Springer's website. The link must be accompanied by the following text: "The final publication is available at link.springer.com". 


\title{
Level sets as progressing waves: an example for wake-free waves in every dimension
}

\author{
Wolfgang Quapp · Josep Maria Bofill
}

Received: 16 September 2013 / Accepted: 31 October 2013 / Published online: 12 November 2013 (C) Springer Science+Business Media New York 2013

\begin{abstract}
The potential energy surface of a molecule can be decomposed into equipotential hypersurfaces of the level sets. It is a foliation. The main result is that the contours are the wave fronts of a certain hyperbolic partial differential equation, a wave equation. It is connected with the gradient lines, as well as with a corresponding eikonal equation. The energy seen as an additional coordinate plays the central role in this treatment. A solution of the wave equation can be a sharp front in the form of a delta distribution. We discuss a general Huygens' principle: there is no wake of the wave solution in every dimension.
\end{abstract}

Keywords Contours - Steepest ascent - Eikonal equation - Wave equation · Huygens' principle

Mathematics Subject Classification $35 \mathrm{~A} 18 \cdot 35 \mathrm{C} 07 \cdot 35 \mathrm{~L} 05$

\footnotetext{
W. Quapp ( $\square)$

Mathematisches Institut, Universität Leipzig, PF 100920, 04009 Leipzig, Germany e-mail: quapp@uni-leipzig.de

URL: www.mathematik.uni-leipzig.de/MI/ quapp

\section{J. M. Bofill}

Departament de Química Orgànica, Universitat de Barcelona,

Martí i Franquès, 1, 08028 Barcelona, Spain

e-mail: jmbofill@ub.edu

\section{J. M. Bofill}

Institut de Química Teòrica i Computacional, Universitat de Barcelona, (IQTCUB),

Martí i Franquès, 1, 08028 Barcelona, Spain
} 


\section{Introduction}

The potential energy surface (PES) [1-3] and the chemical reaction path [4] are the basis for the theories of chemical dynamics. The PES is a continuous function in an $\mathbb{R}^{N+1}$ with respect to the coordinates of the nuclei in an $\mathbb{R}^{N}$, thus it is an $N$ dimensional hypersurface. It should also have continuous derivatives up to a certain order not specified here, but required by the operations which are to be carried out. Of course, the treatment of this paper also holds for any other surface over an $\mathbb{R}^{N}$. The PES is usually an approximaton; firstly the Born-Oppenheimer approximation is done, and then further levels of approximation are used corresponding to the quantum chemical level of interest [5]. Thus the current level of any approximation allows the requirement of a continuous behaviour.

The PES can be seen as formally divided in catchments associated with local minima [1]. The first order saddle points or transition states (TSs) are located at the deepest points of the boundary of the basins. TSs and minima correspond to stationary points of the PES. Two minima of the PES can be connected through a TS via a continuous curve in the $N$-dimensional coordinate space, describing the coordinates of the nuclei. The curve characterizes a reaction path. One can define many types of curves satisfying the above requirement. But the reaction path model mostly used is the steepest descent (SD) from the TS to a reactant or a product. The SD reaction path from TS in mass weighted coordinates is called intrinsic reaction coordinate (IRC) [6-8]. The discussion of a coordinate independent definition of SD curves was already given [9].

Normally, the PES is represented in curvilinear coordinates. One should really calculate with such coordinates; to simplify matters we shall not do so. In this paper we will assume $N$ orthogonal and equidistant coordinates, $\mathbf{q}=\left(q_{1}, \ldots, q_{N}\right)^{T}$, thus Cartesians of the $n$ atoms with $N=3 n$. Then the metric matrix in $\mathbb{R}^{N}$ reduces to the unity matrix.

The SD curves are orthogonal trajectories to the contour hypersurfaces, $V(\mathbf{q})=$ $v=$ constant , if the corresponding metric relations are used [10]. From a theoretical point of view (however not from a numerical one) the SD curves and the inverse ones, the steepest ascent (SA) curves are equivalent. Thus the SA/SD curves emerging from a minimum or a TS of the PES travel in an orthogonal manner through the contour hypersurfaces of this PES. The level sets foliate the PES in a simple kind [11]. It should be noted that the construction of the contour hypersurface, $V(\mathbf{q})=v=$ constant, is such that all points satisfying this equation possess the same equipotential difference with respect to another contour hypersurface. It is similar to the construction of Fermat-Huygens of propagation of rays and wavefronts. This construction and the Hamilton-Jacobi theory are strongly connected [12]. The propagation of level sets with an increase of $v$ seems to develop analogously to the propagation of waves in an inhomogeneous medium with singularities at the stationary points. Thus, to every fixed surface $V(\mathbf{q})$ should exist a corresponding wave equation which allows solutions which are the level sets of $V(\mathbf{q})$. And yes, after we guessed well, we could find such a wave equation (see [13]) which we repeat in Sect. 2. The application to the contour hypersurfaces is given in Sect. 3, and in Sect. 4 we present a splitting formula for the wave operator which may be important for the dimension problem: our solution holds for odd as well as for even dimensions. That is strange for solutions of wave equations. 
In Sect. 5 we treat the wave propagation of level sets. The last section is the discussion of the results.

\section{The wave equation for level sets}

Let $v=V(\mathbf{q})$ be an $N$-dimensional surface in $\mathbb{R}^{N+1}$ of points $\left(q^{1}, \ldots, q^{N}, v\right)$. We treat regions without stationary points of the function $V(\mathbf{q})$. Let $\mathbf{g}(\mathbf{q})$ be the gradient vector, $\nabla_{\mathbf{q}} V(\mathbf{q})$, and let $\mathbf{H}(\mathbf{q})$ be the matrix of its second derivatives, the Hessian $\nabla_{\mathbf{q}} \mathbf{g}^{T}$. We form the scalar product of the gradient with itself. It produces the value $G(\mathbf{q}):=\partial_{\mathbf{q}} V(\mathbf{q})^{T} \partial_{\mathbf{q}} V(\mathbf{q})$. It holds $G(\mathbf{q})>0$. Note that $v$ is the energy if the surface is the PES of a molecule. Let us consider a linear wave operator in $N+1$ dimensions, $q_{1}, \ldots, q_{N}, v[13]$

$$
L:=\left(\nabla_{\mathbf{q}}^{2}-G(\mathbf{q}) \frac{\partial^{2}}{\partial v^{2}}+\operatorname{Trace} \mathbf{H}(\mathbf{q}) \frac{\partial}{\partial v}\right)
$$

where $\nabla_{\mathbf{q}}^{2}=\nabla_{\mathbf{q}}^{T} \nabla_{\mathbf{q}}=\partial^{2} / \partial q_{1}^{2}+\cdots+\partial^{2} / \partial q_{N}^{2}$ is the Laplacian in $\mathbb{R}^{N}$, and Trace $\mathbf{H}$ is the sum of the diagonal entries of $\mathbf{H}$. The operator $L$ is of the hyperbolic type by signature $++\cdots+-$ since $G(\mathbf{q})>0$, thus outside of stationary points of $\mathrm{V}(\mathbf{q})$. We search for a solution of the equation

$$
L \psi(\mathbf{q}, v)=0
$$

The reasoning for the introduction of such an equation will come true with its success.

\section{Theorem}

Be $F$ a function of one real variable, $F: \mathbb{R} \rightarrow \mathbb{R}$, with first and second continuous derivations, then

$$
\psi(\mathbf{q}, v)=F(V(\mathbf{q})-v)
$$

is a solution of the wave Eq. (2).

For other wave equations, one may compare the ansatz with that of Courant/Hilbert [12] p. 620, Friedlander [14] p. 110, Bombelli and Sonego [15], Hillion [16,17], and Duistermaat [18].

The proof of the Theorem is straightforward: computing the differential expression $L \psi$, we get

$$
L \psi=F^{\prime \prime}(. .)\left[\left(\nabla_{\mathbf{q}} V\right)^{T}\left(\nabla_{\mathbf{q}} V\right)-G\right]+F^{\prime}(. .)\left[\nabla_{\mathbf{q}}^{2} V-\text { Trace } \mathbf{H}\right]=0
$$

by the definition of the values of $G$ and Trace $\mathbf{H}$. $F$ can be an arbitrary function, because the two coefficients of $F^{\prime \prime}$ and $F^{\prime}$ are zero independently.

Note

(i) The first coefficient of Eq. (4) is the Hamilton-Jacobi equation or eikonal equation, a partial differential equation of the first order. It describes a relation between the level contours of a surface and its SD curves [19] 


$$
\left(\nabla_{\mathbf{q}} V(\mathbf{q})\right)^{T}\left(\nabla_{\mathbf{q}} V(\mathbf{q})\right)=G(\mathbf{q}) .
$$

Many papers describe the sulution, see [20-22] and references therein. The eikonal also emerges in a variational theory of steepest descent curves [19]. Usually we have to solve it for $V(\mathbf{q})$, if a general function $G(\mathbf{q})$ is given. Here it is automatically fulfilled by the definition of $G(\mathbf{q})$.

(ii) The second coefficient in Eq. (4) is usually named transport equation of the operator $L$ [14], it emerges here in a simple version. It is also automatically zero by the special choice of the operator $L$ with the term $\operatorname{Trace} \mathbf{H}$.

The phase function

$$
S(\mathbf{q}, v)=V(\mathbf{q})-v
$$

fulfills the characteristic equation [12] pertaining to operator $L$ of Eq. (1)

$$
\left(\nabla_{\mathbf{q}} S(\mathbf{q}, v)\right)^{T}\left(\nabla_{\mathbf{q}} S(\mathbf{q}, v)\right)-G(\mathbf{q})\left(\frac{\partial}{\partial v} S(\mathbf{q}, v)\right)^{2}=0 .
$$

It is identical with Eq. (5). Characteristic surfaces $S(\mathbf{q}, v)=0$ are considered as potential carriers of wave fronts [12]. But the hypersurfaces $S(\mathbf{q}, v)=0$ with fixed $v$ are also the contours of the surface. A further trivial solution of system (7) is the function $S_{+}(\mathbf{q}, v)=V(\mathbf{q})+\left(v-2 v_{0}\right)$ with any fixed value $v_{0}$.

Solution (3) indicates initial values for $v=v_{0}$. If we put $D(x)=-F^{\prime}(x)$ we have

$$
\psi\left(\mathbf{q}, v_{o}\right)=F\left(V(\mathbf{q})-v_{o}\right)
$$

and

$$
\left.\frac{\partial}{\partial \nu} \psi(\mathbf{q}, \nu)\right|_{\nu=v_{0}}=D\left(V(\mathbf{q})-v_{o}\right) .
$$

Of course, $D(x)$ is not independent from $F(x)$, thus, (8) and (9) do not pose the most general initial values. Thus, solution (3) would not be the most general solution to the wave Eq. (2) [23]. However, the conditions (8) and (9) are a well posed Cauchy initial value problem for Eq. (2).

To further imagine the first derivative to $v$ we use the identity

$$
\frac{\partial}{\partial v} F(V(\mathbf{q})-v)=(-1) \frac{1}{G(\mathbf{q})} \sum_{i} \frac{\partial V}{\partial q_{i}} \frac{\partial}{\partial q_{i}} F(V(\mathbf{q})-v) .
$$

It means that the change of the solution in the $v$-direction in the space $\mathbb{R}^{N+1}$ corresponds to the directional derivative in the direction of the gradient of $V(\mathbf{q})$ in the coordinate space $\mathbb{R}^{N}$, as one expects it for the level hypersurfaces. On the other hand, we can treat the phase function at its zero level $S(\mathbf{q}, v)=V(\mathbf{q})-v=0$. Let us 
expand $S$ into a Taylor series up to the first order around point $\left(\mathbf{q}_{o}, v_{o}\right)$ on a level set, say, $V\left(\mathbf{q}_{o}\right)-v_{o}=0$.

$$
\begin{aligned}
S(\mathbf{q}, v)= & S\left(\mathbf{q}_{o}, v_{o}\right)+\left.\frac{\partial S\left(\mathbf{q}_{o}, v\right)}{\partial v}\right|_{\nu=v_{o}}\left(v-v_{o}\right) \\
& +\left.\sum_{i}^{N} \frac{\partial S\left(\mathbf{q}, v_{o}\right)}{\partial q_{i}}\right|_{q_{i}=q_{i o}}\left(q_{i}-q_{i o}\right)+\cdots
\end{aligned}
$$

If we assume that the point $(\mathbf{q}, v)$ is on a next level hypersurface as well, with $v>v_{0}$, then we have to the first order

$$
\left.\sum_{i}^{N} \frac{\partial V(\mathbf{q})}{\partial q_{i}}\right|_{q_{i}=q_{i o}} \frac{\left(q_{i}-q_{i o}\right)}{\left(v-v_{o}\right)}=1
$$

since it is $\left.\frac{\partial S(\mathbf{q}, v)}{\partial v}\right|_{\nu=v_{o}}=-1$ and $\left.\frac{\partial S(\mathbf{q}, v)}{\partial q_{i}}\right|_{q_{i}=q_{i o}}=\left.\frac{\partial V(\mathbf{q})}{\partial q_{i}}\right|_{q_{i}=q_{i o}}$ for $i=1, \ldots, N$.

Equation (12) means $\sum_{i} \frac{\partial V(\mathbf{q})}{\partial q_{i}} \frac{d q_{i}}{d v}=\frac{d V(\mathbf{q})}{d \nu}=\frac{d v}{d v}=1$, which is true. However, (12) is also fulfilled if we approximate the $q_{i}$-steps by

$$
\frac{\left(q_{i}-q_{i o}\right)}{\left(v-v_{o}\right)}=\frac{g_{i o}}{G\left(\mathbf{q}_{o}\right)} \text { for } i=1, \ldots, N
$$

Finally, up to the first order the steepest ascent curve as a function of $v$ takes the form [24]

$$
\mathbf{q}(v)=\mathbf{q}\left(v_{o}\right)+\left.\frac{d(\mathbf{q})}{d v}\right|_{q_{i}=q_{i o}}\left(v-v_{o}\right)=\mathbf{q}\left(v_{o}\right)+\frac{\mathbf{g}_{o}}{G\left(\mathbf{q}_{\mathbf{o}}\right)}\left(v-v_{o}\right) .
$$

$\mathbf{q}(v)$ describes the "rays" which connect the contour hypersurfaces (6) with $S=0$. The steepest ascent $\mathbf{q}(v)$ is a curve on the characteristic surface, thus, it is a bicharacteristic in $I R^{N+1}$. The generalized orthogonality between rays and wavefronts is already described [25].

The solution (3) is a progressing wave which is in its general form defined by

$$
\phi(\mathbf{q}, v)=U(\mathbf{q}, v) F(S(\mathbf{q}, v))
$$

where we use scalar functions $U$ and $S$. Here we have the phase function, $S(\mathbf{q}, v)=$ $V(\mathbf{q})-v$, with nowhere vanishing gradient, and the trivial amplitude $U=1$. The formula further contains any wave form or "wave profile" function $F: \mathbb{R} \rightarrow \mathbb{R}$ $[12,14-17,23,26-30]$. Note that solution (3) is simpler than some spherical wave solutions for wave equations with constant coefficients [12,31] because we do not need neither the so-called "distortion"-factor, $U$, nor any derivations of the function $F$ for higher $N$. Solution (3) holds for every dimension $N$, see also below Sect. 4. 


\section{Level hypersurfaces}

In the classical theory, a solution of a differential equation must possess derivatives of all orders that appear in the equation. This requirement is frequently ignored in mathematical chemistry. Here, the wave equation in the $\mathbb{R}^{N+1}$ of 'space-energy' will be considered in the context of distribution theory [14]. We use the special form of a progressing wave of the solution (3) for a special distribution. Let $V(\mathbf{q})$ be a realvalued function on a region of the coordinate space, and let $F(x)$ be a function on $\mathbf{R}$. Then the composite function $F(V(q))=F \circ V(\mathbf{q})$ is a function on the coordinate space. Now we replace the function $F$ of the wave form by the distribution $\delta$, so to say, by a "sharp impulse" with a unit integral. The chain rule holds [14] for every derivation

$$
\frac{\partial}{\partial q_{i}} \delta(V(\mathbf{q}))=\delta^{\prime}(V(\mathbf{q})) \frac{\partial}{\partial q_{i}} V(\mathbf{q})
$$

in the sense of the distribution space. Thus, the proof from Sect. 2 also holds in the limit for distributions.

In Fig. 1 we show a schematic surface for a first understanding of the matter. The starting point is the situation for $N=1$, and $V(q)$ is a Morse potential. If we use solution (3) with function $F(x)=x$ then we get the surface of Fig. 1. The Morse function is 'sandwiched' with increasing $v$ [15]. A compact support of $V(\mathbf{q})$ implies such a 'sandwich propagation'. It is an example of a foliation.

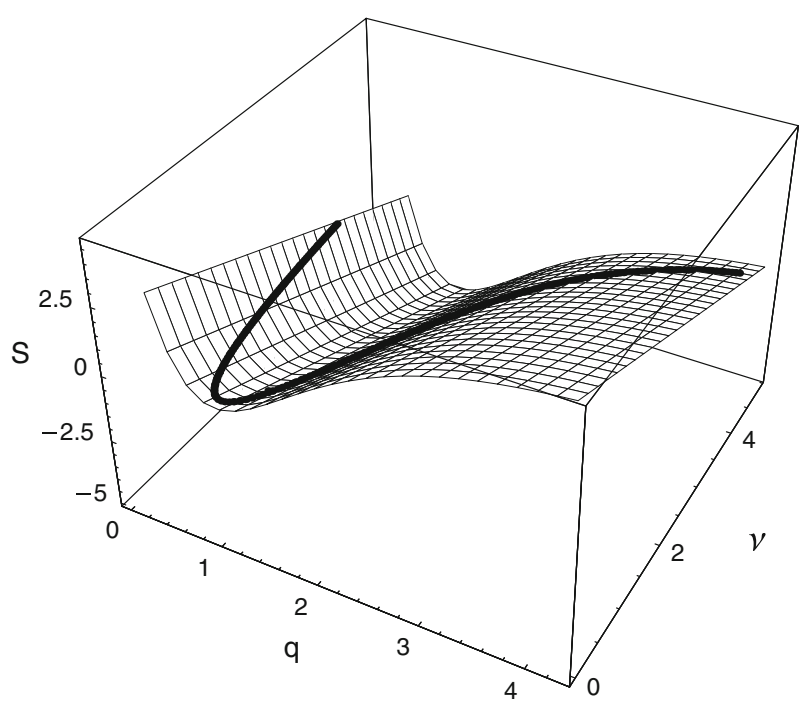

Fig. 1 A one-dimensional Morse function $V(q)$ is used for a picture of the solution surface $\Psi(q, v)=$ $V(q)-v$. Thus, we employ the 'wave form' function $F(x)=x$. In contrast, the bold curve is the intersection with $V(q)=v$, the zero plane of $\Psi=S$. The bold curve is the support of the distribution $\delta(V(q)-v)$ 
However, if we use the distribution limit $F(x) \rightarrow \delta(x)$, thus [29]

$$
\psi(\mathbf{q}, v)=\delta(V(\mathbf{q})-v)
$$

the situation changes dramatically. The solution is zero except at $V(\mathbf{q})=v$, at every level hypersurface-in Fig. 1 it is a point, or there are two points, correspondingly, if $v \geq 0$. We may imagine a given level hypersurface at $v=v_{o}$. It is a source of the wave equation of unit strength [28]. If $v$ proceeds, each point in $(\mathbf{q}, v)$ space in solution (17) results in a zero value except at the level $v=V(\mathbf{q})$ which forms the corresponding level hypersurface. Since we treat regions without stationary points of the function $V(\mathbf{q})$, the function changes monotonically with $v$ (of course this holds automatically per construction because $v$ is the value of $V(\mathbf{q})$ ), we have always a sharp wave front. If the 'impulse' passes the point $(\mathbf{q}, v)$ with $v=V(\mathbf{q})$ then (17) undergoes an impulse displacement of strength 1 . There is no wake; once the wave passes by, the displacement is again zero. We obtain "sharp" level hypersurfaces of $V(\mathbf{q})$. It is a criterion for the validity of a general Huygens' principle [32].

\section{The dimension problem}

The solution (17) does not divide the dimensional cases of odd or even dimensions of the coordinate space. The proof obviously holds for every number of dimensions. This is an astonishing result [12], and it is a hint that the strong Huygens' principle in the sense of Hadamard does not hold here $[33,34]$. We underline it with a partitioning of the coordinate space $I R^{N}$ into two sets of variables $\mathbf{q}_{I}^{T}=\left(q_{1}, \ldots, q_{M}\right)$ and $\mathbf{q}_{I I}^{T}=$ $\left(q_{M+1}, \ldots, q_{N}\right)$ with $1 \leq M<N$. Due to the sum structure of the coefficients $G(\mathbf{q})$ and Trace $\mathbf{H}(\mathbf{q})$ we can split them into

$$
\begin{aligned}
G(\mathbf{q}) & =G\left(\mathbf{q}_{I}\right)+G\left(\mathbf{q}_{I I}\right) \text { and } \\
\text { Trace } \mathbf{H}(\mathbf{q}) & =\text { Trace } \mathbf{H}\left(\mathbf{q}_{I}\right)+\text { Trace } \mathbf{H}\left(\mathbf{q}_{I I}\right) .
\end{aligned}
$$

Then we can write the differential Eq. (2) as

$$
L \psi(\mathbf{q}, v)=L_{I} \psi\left(\mathbf{q}_{I}, \mathbf{q}_{I I}, v\right)+L_{I I} \psi\left(\mathbf{q}_{I}, \mathbf{q}_{I I}, v\right)=0
$$

where the operator $L_{I}$ is

$$
L_{I}:=\left(\nabla_{\mathbf{q}_{I}}^{2}-G\left(\mathbf{q}_{I}\right) \frac{\partial^{2}}{\partial \nu^{2}}+\operatorname{Trace} \mathbf{H}\left(\mathbf{q}_{I}\right) \frac{\partial}{\partial v}\right)
$$

acting in the $I R^{M+1}$, and the same for $L_{I I}$ acting in the $I R^{N-M+1}$. If $\psi\left(\mathbf{q}_{I}, \mathbf{q}_{I I}, v\right)$ is a solution of Eq. (2) then also holds $L_{I} \psi\left(\mathbf{q}_{I}, \mathbf{q}_{I I}, v\right)=-L_{I I} \psi\left(\mathbf{q}_{I}, \mathbf{q}_{I I}, v\right)$. The unique solution is $L_{I} \psi\left(\mathbf{q}_{I}, \mathbf{q}_{I I}, v\right)=L_{I I} \psi\left(\mathbf{q}_{I}, \mathbf{q}_{I I}, v\right)=0$ which is independent of the partitioning. Especially, if $N$ was even, then a splitting into $N=M+(N-M)$ with an odd $M$ produces two 'odd' operators. 
All in all, we can conclude: every "full" level hypersurface, $V(\mathbf{q})=v$, of a surface, $V(\mathbf{q})$, over coordinates in the $\mathbb{I}^{N}$ is an $(N-1)$-hypersurface and fulfills the $(N+1)$-dimensional differential Eq. (2) with solutions (3) or (17); but every restriction to subspaces of dimension $M<N$ forms a section in the subspace of the level hypersurface, of course on the same level $v$, and it fulfills a reduced differential Eq. (21) in that subspace. Thus, the operator $L$ in Eq. (1) can be totally split into single summands

$$
L=\sum_{i=1}^{N}\left(\frac{\partial^{2}}{\partial q_{i}^{2}}-\left(\frac{\partial V}{\partial q_{i}}\right)^{2} \frac{\partial^{2}}{\partial \nu^{2}}+\frac{\partial^{2} V}{\partial q_{i}^{2}} \frac{\partial}{\partial v}\right)=: \sum_{i=1}^{N} L_{i}
$$

and every part $L_{i}$ fulfills $L_{i} \psi=0$ where $\psi$ is a solution of $L \psi=0$. The proof (4) holds for every single $L_{i}$. A case $i=N=1$ is shown in Fig. 1.

\section{Wave propagation}

By the structure of the wave operator (1) we do not need an integration process for the solution (3). Before, we have used the surface $V(\mathbf{q})$ for the construction of the operator $L$ in Eq. (1) taking advantage of its first and second derivations, and have then found the surface $V(\mathbf{q})$ itself in the solution. So, here the usual integrals over conoids do not emerge like in the general theory of wave equations $[12,14,35,36]$. As above we assume that we are in a region between stationary points of the surface, $V(\mathbf{q})$, but the stationary points themselves are excluded, thus it is $G(\mathbf{q})>0$. We ask for the kind of mapping between the different level hypersurfaces which emerge in the solution (17). In Fig. 2 we show the convex level lines of a two-dimensional surface. We know from the wave equation theory that "rays" connect the wave fronts. From the usual behavior of steepest ascent curves, corresponding steepest descent ones, we know that every point of a level line to a fixed level $v=v_{o}$ is one-to-one, or bijectively

Fig. 2 Level lines of a two-dimensional function $V\left(q_{1}, q_{2}\right)$ (thin curves) and a steepest ascent curve (dashes)

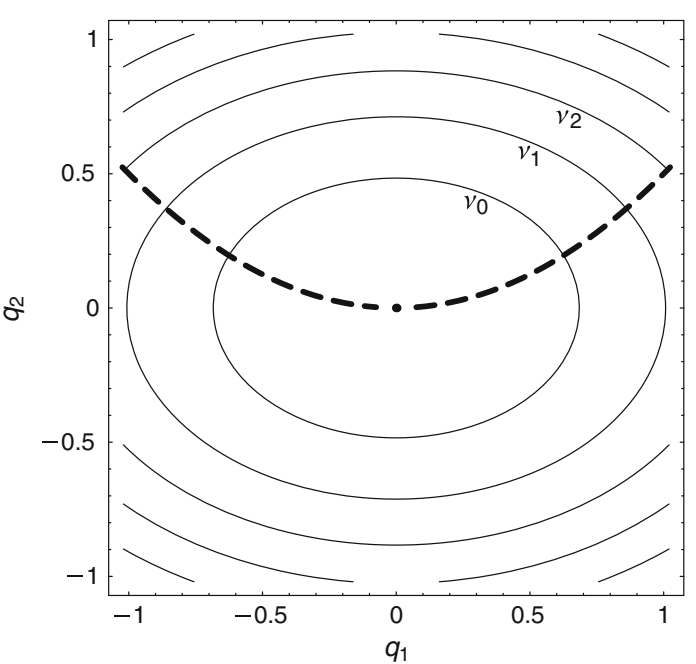


connected to a point of another level line $v=v_{1}$, see Eq. (14) [19]. The family of all steepest ascent curves is a mapping of the level lines, $A\left(v_{0}\right) \rightarrow A(v)$ where $A\left(v_{0}\right)$ is the set of points $\left(\mathbf{q}, v_{0}\right)$ which fulfills $V(\mathbf{q})=v_{0}$. It holds

$$
A\left(v_{0}\right) \rightarrow A\left(v_{2}\right)=A\left(v_{0}\right) \rightarrow A\left(v_{1}\right) \text { o } A\left(v_{1}\right) \rightarrow A\left(v_{2}\right)
$$

if $v_{0}<v_{1}<v_{2}$, because the corresponding relation holds on every curve of the family of steepest ascent curves. (It holds: the mapping is a semi group.) From this point of view we do not deal with forward- and backward conoids of influence, like in the theory of wave equations, but only with one-dimensional curves, the steepest ascent, or steepest descent curves [13]. In the treated regions where $G(\mathbf{q})>0$, it holds however, that all solutions (17) are "sharp" hypersurfaces. So to say, level hypersurfaces are "sharp" wavefronts per definition. They are "sharp" sections with the plane $v=$ constant. It means that they fulfill a general Huygens's principle of propagation.

It is known [12] that the solutions of the ordinary wave equation (with constant coefficients) in odd space dimensions $(N \geq 3)$ possess basic differences from those in even dimensions. A physical conclusion is: If a source signal ceases, the disturbance at a finitely distanced point will also cease in a finite time, i.e., sharp signals remain sharp. This can only occur in an odd dimension. In contrast, in even dimensions, an expanding wave does not have this property; there is an indefinitely prolonged tail. Thus, traveling waves resulting from general localized sources in odd space dimensions are sharp, while those in even space dimensions are not. This is a short form of Hadamards minor premise of the strong Huygens' principle [33,34], and one can conjecture that this overall situation also may pertain for wave equations with non constant coefficients $[12,14,35,36]$, especially that in even space dimensions a strong Huygens' principle does not hold.

In our case, where the physical time is replaced by the energy, $v$, the hyperbolic Eq. (2) is defined in an inhomogeneous medium, caused by the coefficient $G(\mathbf{q})$, and it has a wake-free expanding wave solution (17), which is unattenuated, and whose wave front propagates up to energies-where a useful PES region ends. In contrast to the Hadamard conjecture above, the solution (17) is independent of odd/even space dimensions. It follows that the hyperbolic wave Eq. (2) is not a Huygens' differential equation in the sence of Hadamard [33].

\section{Discussion}

The present investigation can be regarded as an investigation of operators that allow undistorted progressing wave families as defined by Courant [12]. The wave front (17) solves the differential equation (2) in an $\mathbb{R}^{N+1}$. It satisfies a general Huygens' principle corresponding to its distributional character throughout. By a general Huygens' principle of the propagation of waves we understand that sharp signals propagate as sharp signals. The "signal" here is the $(N-1)$-contour to a corresponding level of a given surface over an $\mathbb{R}^{N}$. The differential equation (2) is linear, but its second order part has one non constant coefficient. Its solution (17) is a special progressing wave in a very simple form, which does not divide the dimensional cases of odd or 
even dimensions of the coordinate space. This is an astonishing result. We exclude that Eq. (2) itself is a Huygens' equation by its dimensional behaviour. Additionally, we do not treat with conditions (8) and (9) the most general initial value problem [37]. Nevertheless, it is perhaps worth pointing out that operator $L$ in Eq. (1) allows undistorted progressing waves in every dimension, and its splitting property (22) is a hint that we could bring forward a general Huygens' principle of the propagation of level hypersurfaces from one kind of subdimension $M<N$, say odd, to the other kind, say even, and vice versa.

Acknowledgments Financial support from the Spanish Ministerio de Ciencia e Innovación, DGI project CTQ2011-22505 and, in part from the Generalitat de Catalunya projects 2009SGR-1472 is fully acknowledged. It is a pleasure to thank Prof. R. Schimming for a helpful discussion to Ref. [13]. We thank M. Belger for a careful reading of the manuscript.

\section{References}

1. P.G. Mezey, Potential Energy Hypersurfaces (Elsevier, Amsterdam, 1987)

2. D. Heidrich, W. Kliesch, W. Quapp, Properties of Chemical Interesting Potential Energy Surfaces, Lecture Notes Chemistry 56 (Springer, Berlin, 1991)

3. D.J. Wales, Energy Landscapes (Cambridge Univ. Press, Cambridge, 2003)

4. D. Heidrich (ed.), The Reaction Path in Chemistry: Current Approaches and Perspectives (Kluwer, Dordrecht, 1995)

5. W. Kutzelnigg, Einführung in die Theoretische Chemie, vol. 2 (VCH, Weinheim, 1994)

6. K. Fukui, J. Phys. Chem. 74, 416 (1970)

7. A. Tachibana, K. Fukui, Theor. Chim. Acta 49, 321 (1978)

8. A. Tachibana, K. Fukui, Theor. Chim. Acta 51(189), 275 (1979)

9. W. Quapp, D. Heidrich, Theor. Chim. Acta 66, 245 (1984)

10. W. Quapp, J. Theor. Comput. Chem. 2, 385 (2003)

11. A. Candel, L. Colon, Foliations I (AMS, Providence, 2000)

12. R. Courant, D. Hilbert, Methods of Mathematical Physics Part 2 (by R. Courant), 2 ed, English ed., (Wiley: New York, 1953)

13. J.M. Bofill, W. Quapp, M. Caballero, J. Chem. Theor. Comput. 8, 4856 (2012)

14. F.G. Friedlander, The Wave Equation on a Curved Space-Time (Cambridge Univ. Press, London, 1975)

15. L. Bombelli, S. Sonego, J. Phys. A 27, 7177 (1994)

16. P. Hillion, J. Math. Phys. 33, 2749 (1992)

17. P. Hillion, Europhys. Lett. 33, 7 (1996)

18. J.J. Duistermaat, Symplectic Geometry (Uni. Utrecht, preprint, 2004)

19. J.M. Bofill, R. Crehuet, J. Chem. Phys. 122, 234105 (2005)

20. T. Cecil, J. Qian, S. Osher, J. Comput. Phys. 196, 327-347 (2004)

21. S. Osher, J.A. Sethian, J. Comput. Phys. 79, 12-14 (1988)

22. J.A. Sethian, Level Set Methods and Fast Marching Methods (Cambridge Univ Press, Cambridge, 1999)

23. H.P.W. Gottlieb, J. Math. Phys. 29, 2434 (1988)

24. A. Aguilar-Mogas, X. Giménez, J.M. Bofill, J. Chem. Phys. 128, 104102 (2008)

25. I. Bucataru, M.A. Slawinski, Nonlin. Anal. Real World Appl. 6, 211 (2005)

26. W.E. Couch, R.J. Torrence, Phys. Lett. A 117, 270 (1986)

27. L. Bombelli, W.E. Couch, R.J. Torrence, J. Math. Phys. 32, 106 (1991)

28. H. Soodak, M.S. Tiersten, Am. J. Phys. 61, 395 (1993)

29. R.S. Ward, Class. Quantum Grav. 4, 775 (1987)

30. D. Baskin, R. Mazzeo, Some Aspects of Linear Wave Equations 2012 (CMI/ETH Summer School 2008)

31. S.L. Sobolev, Some Applications of Functional Analysis in Mathematical Physics (AMS, 1963), Sect. 18.2

32. Ch. Huygens, Traité de la Lumière (Pierre van der Aa: Leiden, 1690); in German: Abhandlung über das Licht (Harry Deutsch Verl.: Frankfurt, 1996)—Ostwalds Klassiker Bd.20 
33. J. Hadamard, Lectures on Cauchy's Problem in Linear Partial Differential Equations (Oxford Univ. Press, London, 1923)

34. Y. Berest, Acta Appl. Math. 53, 125 (1998)

35. P. Günther, Huygens' Principle and Hyperbolic Equations (Academic Press, Boston, 1988)

36. P. Günther, Math. Intell. 13, 56 (1991)

37. A.P. Veselov, Huygens' principle, in Encyclopedia of Nonlinear Science, ed. by A. Scott (Routledge, London, 2004) 